\title{
TARSKI'S INFLUENCE ON COMPUTER SCIENCE
}

\author{
SOLOMON FEFERMAN
}

Departments of Mathematics and Philosophy, Stanford University e-mail address: sf@csli.stanford.edu

\begin{abstract}
Alfred Tarski's influence on computer science was indirect but significant in a number of directions and was in certain respects fundamental. Here surveyed is Tarski's work on the decision procedure for algebra and geometry, the method of elimination of quantifiers, the semantics of formal languages, model-theoretic preservation theorems, and algebraic logic; various connections of each with computer science are taken up.
\end{abstract}

The following is the text of an invited lecture for the LICS 2005 meeting held in Chicago June 26-29, 2005. ${ }^{1}$

Almost exactly eight years ago today, Anita Feferman gave a lecture for LICS 1997 at the University of Warsaw with the title, "The saga of Alfred Tarski: From Warsaw to Berkeley." Anita used the opportunity to tell various things we had learned about Tarski while working on our biography of him. We had no idea then how long it would take to finish that work; it was finally completed in 2004 and appeared in the fall of that year under the title, Alfred Tarski: Life and Logic [14. The saga that Anita recounted took Tarski from the beginning of the $20^{\text {th }}$ century with his birth to a middle-class Jewish family and upbringing in Warsaw, through his university studies and Ph.D. at the ripe young age of 23 and on to his rise as the premier logician in Poland in the 1930s and increasing visibility on the international scene-despite which he never succeeded in obtaining a chair as professor to match his achievements. The saga continued with Tarski coming to Harvard for a meeting in early September, 1939 when the Nazis invaded Poland on September 1st, at which point he was, in effect, stranded. Then, during the next few years he went from one temporary research or teaching position to another on the East Coast. He was finally offered a one year position in 1942 as Lecturer in Mathematics at the then far off University of California in Berkeley, with the suggestion that it might stretch into something longer. In fact, he not

2000 ACM Subject Classification: F.4.1, F.4.3.

Key words and phrases: Tarski, decision procedures, quantifier elimination, cylindrical algebraic decomposition (CAD), time complexity, space complexity, semantics for formal languages, fixed point theorem, finite model theory, preservation theorems, relational database theory, algebraic logic, relation algebras, cylindric algebras,

${ }^{1}$ I want to thank the organizers of LICS 2005 for inviting me to give this lecture and for suggesting the topic of Tarski's influence on computer science, a timely suggestion for several reasons. I appreciate the assistance of Deian Tabakov and Shawn Standefer in preparing the LATEXversion of this text. Except for the addition of references, footnotes, corrections of a few points and stylistic changes, the text is essentially as delivered. Subsequent to the lecture I received interesting comments from several colleagues that would have led me to expand on some of the topics as well as the list of references, had I had the time to do so. 
only succeeded in staying, but rose to the rank of Associate Professor by the end of the war and a year later was made Full Professor, thus finally obtaining the position he deserved. At Berkeley, Tarski built from scratch one of the world's leading centers in mathematical logic, and he remained there, working intensively with students, colleagues and visitors until his death in 1983.

Tarski became recognized as one of the most important logicians of the $20^{\text {th }}$ century through his many contributions to the areas of set theory, model theory, the semantics of formal languages, decidable theories and decision procedures, undecidable theories, universal algebra, axiomatics of geometry, and algebraic logic. What, in all that, are the connections with computer science? When Anita started working on the biography-which only later became a joint project-she asked me and some of my colleagues exactly that question, and my response was: none. In contrast to that-as she said at the conclusion of her Warsaw lecture-John Etchemendy (my colleague in Philosophy at Stanford, and now the Provost of the University) responded: "You see those big shiny Oracle towers on Highway 101? They would never have been built without Tarski's work on the recursive definitions of satisfaction and truth." ${ }^{2}$ It took me a while to see in what sense that was right. Indeed-as I was to learn-there is much, much more to say about his influence on computer science, and that's the subject of my talk today. I owe a lot to a number of colleagues in the logic and computer science areas for pointing me in the right directions in which to pursue this and also for providing me with very helpful specific information. ${ }^{3}$

Alfred Tarski: Life and Logic was written for a general audience; the biographical material is interspersed with interludes that try to give a substantive yet accessible idea of Tarski's main accomplishments. Still, given the kind of book it is, we could not go into great detail about his achievements, and in particular could only touch on the relationship of his work to computer science. Before enlarging on that subject now, I want to tell a story that is in our biography ([14, p. 220-230), and is in many respects revelatory of his own attitude towards the connection.

I had the good fortune to be Tarski's student in the 1950s when he was beginning the systematic development of model theory and algebras of logic. In 1957, the year that I finished my Ph.D., a month-long Summer Institute in Symbolic Logic was held at Cornell University. That proved to be a legendary meeting; in the words of Anil Nerode: "There has been nothing else in logic remotely comparable." What the Cornell conference did was to bring together for the first time, leaders, up-and-coming researchers, and students in all the main areas of logic, namely model theory, set theory, recursion theory, and proof theory. Besides Tarski, the top people there-along with their coteries-were Alonzo Church, Stephen Kleene, Willard Quine, Barkley Rosser, and, in the next generation, Abraham Robinson and George Kreisel. The organization of the meeting itself had been inspired by the mathematician Paul Halmos, who, independently of Tarski, had developed another approach to

\footnotetext{
${ }^{2}$ For those who may not know what the "big shiny Oracle towers" are, the reference is to the headquarters of Oracle Corporation on the Redwood Shores area of the San Francisco Peninsula. A duly shiny photograph of a few of these towers may be found at

http://en.wikipedia.org/wiki/Image:Oracle_Corporation_HQ.png.

${ }^{3} \mathrm{I}$ am most indebted in this respect to Phokion Kolaitis. Besides him I have also received useful comments from Michael Beeson, Bruno Buchberger, George Collins, John Etchemendy, Donald Knuth, Janos Makowsky, Victor Marek, Ursula Martin, John Mitchell, Vaughan Pratt, Natarajan Shankar, and Adam Strzebonski. And finally, I would like to thank the two anonymous referees for a number of helpful corrections.
} 
the algebra of first-order logic. As Halmos wrote about it in his Automathography [23], p. 215:

There weren't many conferences, jamborees, colloquia in those days and the few that existed were treasured. .... I decided it would be nice to have one in logic, particularly if it were at least partly algebraic.

And, "nice" it was.

In addition to the four main areas, the Cornell logic conference was the first to include many speakers from the emerging field of computer science, the theoretical foundations of which had been laid in the 30s by Gödel, Church, Turing, Post, and Kleene. The connections between the theory and application of computation began toward the end of World War II when the first large scale electronic digital computers were built. At that point, for each kind of application, the hardware had to be programmed by hand, a long and arduous task. John von Neumann was instrumental in demonstrating how to circumvent that process by introducing the first form of software.

By 1957, companies such as IBM and Remington Rand were producing the first generation of commercial electronic computers, and the high-level programming language FORTRAN had become established as an industry standard. Some-but by no means alllogicians were quick to grasp the implications of these developments. At the Cornell meeting, Rosser gave a talk on the relation between Turing machines and actual computers; Church gave a series of talks on the logical synthesis of switching circuits for computer hardware; and Abraham Robinson spoke on theorem proving as done by man and machine. Among the younger contributors, Michael Rabin and Dana Scott spoke about finite automata, and Martin Davis talked about his implementation on the "Johnniac" computer (at the Institute for Advanced Study) of a decision procedure for the arithmetic of the integers under addition-a procedure that had been discovered in 1930 by Tarski's student Mojzesz Presburger in his Warsaw seminar.

On the industry side, IBM and some of the other companies employed a number of researchers with backgrounds in mathematics and logic, and these people turned out in large numbers at Cornell, both to listen and to speak. There were fifteen talks given by researchers from IBM, many of them demonstrating the utility of FORTRAN-like programs for solving problems of potential interest to logicians. In particular, the talk by George Collins-a former student of Rosser's-on the implementation of parts of Tarski's decision procedure for the algebra of real numbers on an IBM 704 should have caught Tarski's attention because it suggested possible practical applications of his procedure. But a few years ago, when I asked Collins about Tarski's reaction to that, he said: "He didn't show any appreciation for my work, either then or later. I was somewhat surprised and disappointed." It is indeed surprising that-despite Tarski's own recognition of the importance and systematic pursuit of the decision problem for various algebraic theories, he did not evince the least bit of interest in the practical computational applications of those problems for which a decision procedure had been found. And, he didn't even seem to be interested either in the work of Rabin and Scott, both of whom were high on his list of favorites, Scott as a former student and collaborator, and Rabin as someone he wanted to bring to Berkeley.

Let's look at what Tarski and Collins were up to in more detail. At heart, Tarski's decision procedure rests on the solution of an algebraic problem for the reals, i.e., the ordered field $\langle\mathbb{R},+, \times,<, 0,1\rangle$. Tarski's procedure uses the method of elimination of quantifiers to associate with each first-order formula of the language of the reals an equivalent quantifierfree formula; those without free variables are then easily decided. The procedure reduces 
to determining for each system $P$ of polynomial equations and inequalities and one of its variables $x$, whether or not there exists a common real solution $x$; the answer is to be expressed in terms of the coefficients of the polynomials involved and the remaining variables. Algorithms for special cases of this problem go back through the history of algebra. Tarski's procedure generalizes one due to Sturm for computing the number of roots of a real polynomial in a given interval. On the face of it, Tarski's procedure is nonelementary in time complexity, i.e., greater than all finite towers of powers of 2 , and so it was important for Collins to find a more feasible procedure than the one that he had talked about at the 1957 Cornell meeting. Further improvements on Tarski's procedure by Abraham Seidenberg and later by Paul Cohen didn't really help much in that respect. In the meantime, Collins was working on various aspects of computer algebra and in 1974 and 1975 he published 9], 10] a new method of doubly exponential upper bound complexity called Cylindrical Algebraic Decomposition (CAD). Incidentally, this was during a year that he was visiting Stanford University from Wisconsin. ${ }^{4}$

The most comprehensive source of information on the development of the CAD procedure and related work is $\left[7 .{ }^{5}\right.$ Here are a few of the things I learned from that invaluable volume. In the first stage pursuant to quantifier elimination, the CAD algorithm takes all the polynomials in the matrix of a prenex formula $\varphi$ with a total of $m$ free and bound variables, and outputs a cell decomposition in $\mathbb{R}^{m}$, on each cell of which each of the given polynomials is sign invariant; furthermore, the cells are arranged in cylinders. The QE part of the algorithm uses the output of the CAD algorithm to determine which cells of the decomposition satisfy the matrix of $\varphi$ in order to eliminate the bound variables. The first implementation of the CAD method was made in 1979-80 by Collins' student Dennis S. Arnon. In 1991, Collins and another of his students, Hoon Hong, published a substantial improvement for various examples in practice, though not in complexity upper bound, requiring only partial CAD [11. This was subsequently implemented by Hong under the acronym QEPCAD. The Caviness and Johnson volume presents a number of applications, including polynomial optimization, polynomial best approximation in lower degree (by $n-2$ degree polynomials), the topology of semi-algebraic sets, algebraic curve display, and robot motion planning. By the way, the system Mathematica implements another form of CAD, according to Adam Strzebonski of Wolfram Research, Inc.

To round out the complexity picture, Fischer and Rabin [17 gave an EXPTIME lower bound of the form $2^{c n}$ for deciding for sentences of length $n$ whether or not they are true in the reals, no matter what algorithm is used; the same applies even with nondeterministic algorithms, such as via proof systems. They also showed that the cut-point by which EXPTIME sets in, i.e., the least $n_{0}$ such that for all inputs of length $n \geq n_{0}$, at least $2^{c n}$ steps are needed, is not larger than the length of the given algorithm or axiom system for proofs. Thus real algebra is definitely infeasible on sufficiently large, though not exceptionally large inputs. The applications mentioned above are in a gray area with relatively small numbers of variables, where feasibility in practice depends on the specific nature of the problems dealt with. As for space complexity, there is a PSPACE lower bound on the theory of the reals, as a consequence of a result of Stockmeyer's. Ben-Or, Kozen and

\footnotetext{
${ }^{4}$ Collins reports ( 7 , p. 86) a communication from Leonard Monk in 1974 stating that he and Bob Solovay had obtained a triply exponential upper bound decision procedure for real algebra, though not a quantifier elimination procedure. Fischer and Rabin say (op. cit., p. 124) that Solovay found a doubly exponential upper bound, based on Monk's work.

${ }^{5}$ This includes a reprint of Tarski's "A decision method for elementary algebra and geometry" [39].
} 
Reif [4] established an EXPSPACE upper bound and conjectured that the set of true firstorder sentences of the reals is EXPSPACE-complete. The exact time and space complexities of this set is to this date an open problem (Phokion Kolaitis, personal communication). ${ }^{6}$

Tarski's own route to the decision problem for the reals began in the mid-1920s with his development of an elegant first-order axiomatization of geometry [44. One of his main goals was to prove the completeness of this axiomatization, and that led him to consider its interpretation in the first-order theory of the reals. Tarski recognized that the method of eliminating quantifiers that had been initiated by Leopold Löwenheim and then applied by Thoralf Skolem and C. H. Langford was-when it succeeded-a way of determining all the complete extensions of a first-order axiom system-and in particular of proving the completeness of complete systems. In the latter part of the twenties Tarski ran the "exercise sessions" for the seminar at Warsaw University led by the logic professor, Jan Lukasiewicz, and he used the opportunity to systematically pursue the method of elimination of quantifiers. As an "exercise", Tarski suggested to one of the students, Mojzesz Presburger, that he find an elimination-of-quantifiers procedure for the additive theory of natural numbers, i.e., for the structure $\langle\mathbb{N},+,<, 0,1\rangle$. In that case, full quantifier-elimination is not possible, but can be carried out in a definitional extension of its language, obtained by adding as atomic formulas all those of the form $x \equiv y(\bmod m)$ for each $m=2,3,4, \ldots$. Mathematically, the procedure comes down to solving a system of simultaneous congruences and thus the Chinese remainder theorem. Presburger's result served as his master's thesis in 1928 and it was published a year later [33. This slim paper of nine pages was to be his sole work in logic; after that he went to work in the insurance industry. Some people think Presburger should have received the Ph.D. for that work, but it has to be admitted that its significance was not realized until much later. ${ }^{7}$

The set of first-order truths of the additive structure of natural numbers is called Presburger Arithmetic. As I mentioned earlier, Martin Davis presented his work on programming the Presburger procedure on the Johnniac at the Cornell conference in 1957. That was long before Fischer and Rabin [17] showed that there is a doubly-exponential time lower bound on any algorithmic procedure for Presburger Arithmetic, including non-deterministic ones. If Martin had known that, he might not even have tried, even with today's computers. $^{8}$ On the other hand, such lower bounds tell us little about the feasibility in practice of deciding relatively short statements. As to upper bounds, Presburger's own procedure is non-elementary; this was improved to triply-exponential by Derek Oppen 32. A search on "Google Scholar" came up with a number of references to Presburger Arithmetic. Near the top are applications to the symbolic model checking of infinite state systems [5] and proving safety properties of infinite state systems [20]; further applications via combination decision procedures are indicated in [37.

Let's return to Tarski's own work on elimination of quantifiers for the elementary (i.e., first-order) theory of real numbers: although it was obtained by 1930 and he considered

\footnotetext{
${ }^{6}$ Just minutes before my lecture, I learned from Prakash Panangaden that John Canny (U.C. Berkeley School of Engineering) proved [6] that the existential theory of the reals is in PSPACE.

${ }^{7} \mathrm{~A}$ sad coda to this story is that Presburger, a Jew, perished in the Holocaust in 1943.

${ }^{8}$ Shankar 37 takes as an epigram a quote from Davis 12 re his experiment with Presburger Arithmetic: "Its great triumph was to prove that the sum of two even numbers is even." A second epigram from the same source, quoting Hao Wang, is that: "The most interesting lesson from these results is perhaps that even in a fairly rich domain, the theorems actually proved are mostly ones which call on a very small portion of the available resources..."
} 
it to be one of his two most important results (the other being his theory of truth), it's surprising he didn't get around to preparing it for publication until 1939. That was under the title, "The completeness of elementary algebra and geometry" ${ }^{9}$ for a new series on metamathematics planned by a Parisian publisher, but the actual publication was disrupted by the German invasion of France in 1940. As Tarski later wrote: "Two sets of page proofs which are in my possession seem to be the only material remainders of that venture." The next time he got around to working on its publication was in 1948 when his friend and colleague J. C. C. McKinsey was at RAND Corporation in Santa Monica. My guess is that McKinsey suggested to his superiors that there would be potential value to applying Tarski's procedure to the computer calculation of optimal strategies in certain games. (Game theory was in those years a very popular subject at RAND.) However, any implementation would first require writing up its theoretical details in full. Working under Tarski's supervision, McKinsey took on the job, revising the 1939 manuscript in its entirety. That came out as a RAND Report under the new title "A decision procedure for elementary algebra and geometry" in 1948; it was finally brought out publicly three years later by UC Press as a second edition [39]. The change in title from 1939 to 1948/1951 corresponds to a change in aims, from completeness to decidability. (By the way, a lightly edited version of the 1939 page proofs eventually appeared under the original title in 1967 in France).

Though Tarski may not have been interested in actual computation at any time in this entire history, he was interested in mathematical applications of his procedure. In fact, one of Tarski's strongest motivations throughout his career was to attract mathematicians to the results of work in logic, and he often did this by reformulating the results in a way that he thought would be more digestible by mathematicians. One side result he noticed about his elimination-of-quantifiers argument for the first-order theory of the real numbers is that every definable set has the form of a union of a finite number of intervals (not necessarily proper) with algebraic end-points. He used this to illustrate the general concept of definable set of elements in a structure. At the outset of his 1931 paper on definable sets of real numbers [42] he said that mathematicians in general don't like to deal with the notion of definability. One reason is that used informally it can lead to contradictions, like the paradox of Richard; that uses an enumeration in English (say) of all the real numbers definable in English, to define (in English) a real number not in that enumeration, by diagonalization. Another reason for mathematicians' aversion mentioned by Tarski is that mathematicians think the notion of definability is not really part of mathematics. In a way, he agrees, for he says that

The problems of making [the meaning of definability] more precise, of removing the confusions and misunderstandings connected with it, and of establishing its fundamental properties belong to another branch of science-metamathematics. [Italics mine]

In fact, he says, he has "found a general method which allows us to construct a rigorous metamathematical definition of this notion".

But then

by analyzing the definition thus obtained it proves to be possible... to replace it by [one] formulated exclusively in mathematical terms. Under this new definition the notion of definability does not differ from other mathematical notions and need not arouse either fears or doubts; it can be discussed entirely within the domain of normal mathematical reasoning. [Italics mine]

\footnotetext{
${ }^{9}$ By the elementary theory of a structure, Tarski means the set of its first-order truths.
} 
The metamathematical explanation of definability in Tarski's 1931 paper is given in terms of the notion of satisfaction, whose definition is only indicated there. Under the mathematical definition, on the other hand, the definable sets and relations are simply those generated from certain primitive sets of finite sequences corresponding to the atomic formulas, by means of the Boolean operations and the operation of projection. Although the 1931 paper concentrates on the concept of definability in a structure, in a footnote to the metamathematical explanation it is stated that "an analogous method can be successfully applied to define other concepts in the field of metamathematics, e.g., that of true sentence..."

Tarski later spelled this out in his famous 1935 paper "Der Wahrheitsbegriff in den formalisierten Sprachen" (The concept of truth in formalized languages, [41]). ${ }^{10}$ Some regard this work as one of the most important instances of conceptual analysis in twentieth century logic, while others think he was merely belaboring the obvious. After all, logicians like Löwenheim, Skolem and Gödel had been confidently using the notions of satisfaction and truth in a structure in an informal sense for years before Tarski's work and an explicit definition was not deemed to be necessary, unlike, for example, the conceptual analysis of computability by Turing. I have to agree that there is some justice to the criticism since the definitions are practically forced on us, once one attends to providing them at all. But even if that's granted, Tarski's explication of these concepts has proved to be important as a paradigm for all the work in recent years on the semantics of a great variety of formal languages.

In particular, the influence of Tarski on the semantics of programming languages is so pervasive that to detail it would require an entire presentation in itself. Let me mention just one example, namely that of the semantics of the lambda calculus and its extensions via domain theory, as developed by Dana Scott and his followers. This happens to connect with the item in Tarski's list of publications that is most cited in the computer science literature, namely his lattice-theoretic fixpoint theorem [40, which is an elegant abstract formulation of the essential characteristic of definition by recursion. ${ }^{11}$ There is also a significant personal connection: Scott began his studies in logic at Berkeley in the early 50s while still an undergraduate. His unusual abilities were soon recognized and he quickly moved on to graduate classes and seminars with Tarski and became part of the group that surrounded him, including me and Richard Montague; so it was at that time that we became friends. Scott was clearly in line to do a Ph. D. with Tarski, but they had a falling out for reasons explained in our biography of Tarski 14. Upset by that, Scott left for Princeton where he finished with a Ph.D. under Alonzo Church. But it was not long before the relationship between them was mended to the point that Tarski could say to him, "I hope I can call you my student," and rightly so: not only did Scott's thesis deal with a problem that had been proposed by Tarski, but all of Scott's work is in the best Tarskian tradition of breadth, rigor, clarity of exposition and clarity of purpose. And, like Tarski, he prefers settheoretic and algebraic methods, of which the domain-theoretic approach to the semantics

\footnotetext{
${ }^{10}$ It was not until 1957, in a paper with Robert L. Vaught 45], that Tarski explicitly presented these notions as those of satisfaction and truth in a structure. See the discussion by Hodges [26] and Feferman 15] of the relationship of that to the "Wahrheitsbegriff" paper.

${ }^{11}$ Tarski proved that every monotonic function over a complete lattice has a complete lattice of fixed points, and hence a least fixed point. This is a generalization of a much earlier joint result of Knaster and Tarski and so is sometimes referred to as the Knaster-Tarski theorem. A related result used in applications is that every continuous function on a complete lattice has a least fixed point; credit for it is unclear, and thus it is considered a "folk theorem". The history of these and other fixed point theorems relevant to computer science is surveyed in [29].
} 
of type-free functional programming languages is a perfect example. So Tarski's influence on computer science manifests itself here at just one remove, though of course Scott's contribution, beginning in 1976 [36] with the construction of a domain $D$ isomorphic to $D \rightarrow D$, is completely novel. ${ }^{12}$

Satisfaction and truth in a structure are the basic notions of model theory, whose systematic development in the 1950s is initially largely due to Tarski and his school. The notions are relative to a formal language, which is usually taken to be first-order (FO), because of the many happy properties of FO logic such as that of compactness (cf. the texts by Chang and Keisler or Hodges). But other kinds of logics in which, e.g., compactness fails, turned out to be partially susceptible to useful model-theoretic methods as shown by the greatly varied contributions to the collection Model-Theoretic Logics [3. Among these are the model-theory of infinitary languages as well as second-order and higher order languages. For computer science, a great variety of finite structures, such as various classes of graphs, arise naturally, and it was discovered that a number of questions in complexity theory may be framed as questions in finite model theory (cf., e.g. [13]). In addition to first-order logic (FOL) and its finite variable fragments, other logics that have proved to be useful in finite model theory are finite-variable infinitary logics, monadic second-order logic (MSOL) and its fragments, and certain fixed point logics such as Datalog and least fixed-point logic LFP.

Recently there has been a surge of very interesting work on analogues in finite FO model theory to a class of general results called preservation theorems in classical FO model theory. The newest and most exciting of these is due to Ben Rossman 35. So I can limit myself to explaining the general nature of the main results.

Given a relation $R$ between structures and a sentence $\varphi$, we say that $\varphi$ is preserved under $R$ if whenever $M$ satisfies $\varphi$ and $N$ is in the relation $R$ to $M$, then $N$ satisfies $\varphi$. The results from classical FO model theory characterize up to logical equivalence, the form of sentences preserved under various $R$. The most famous ones are the following, all from the 1950 s.

- EPT (Los-Tarski). $\varphi$ is preserved under extensions iff $\varphi$ is equivalent to an existential sentence.

- OHPT (Lyndon). $\varphi$ is preserved under onto-homomorphisms iff $\varphi$ is equivalent to a positive sentence.

- HPT (Łos-Tarski-Lyndon). $\varphi$ is preserved under (into-)homomorphisms iff $\varphi$ is equivalent to an existential positive sentence.

The finite analogues of these results are obtained by restricting to finite $M$ and $N$. The 'if' directions of course hold in all the finite versions, but the 'only if' analogues of the Extension Preservation Theorem (EPT) and the Onto-Homomorphism Preservation Theorem (OHPT) are known to fail. In particular, the failure of the finite analogue of EPT is due to Bill Tait in 1959, who thereby disproved a conjecture of Scott and Suppes; Tait's result was rediscovered by Gurevich and Shelah in 1984. The failure of OHPT in the finite is due to Rosen [34. ${ }^{13}$ What Rossman [35] has proved, surprisingly, is that HPT holds in the finite. There are interesting relations to Datalog programs, which are given by

\footnotetext{
${ }^{12}$ Scott informed me that his use of lattice fixed points was initiated in the fall of 1969 in work with Christopher Strachey and exposed in many lectures in Oxford while on leave there. For further developments and a large bibliography see [21].

${ }^{13}$ Lyndon's famous positivity theorem implies OHPT. Ajtai and Gurevich, and then Stolboushkin in a simpler way, proved failure in the finite of the positivity theorem, but their constructions did not prove failure in the finite of OHPT.
} 
existential positive FO inductive definitions. A Datalog formula may thus be considered as an infinitary disjunction of existential positive FO formulas. Using a simple compactness argument, Rossman's result about HPT in the finite also implies the theorem of Ajtai and Gurevich [1] that on finite structures, if a Datalog sentence is equivalent to a FO sentence then it is equivalent to a single existential positive sentence, exactly those preserved by homomorphisms. ${ }^{14}$ The failure of the Eos-Tarski characterization in the finite shows that preservation theorems do not in general relativize from a class to a subclass. Thus it is also of interest to ask for which classes of finite structures HPT holds. This had already been investigated by Atserias, Dawar and Kolaitis 2] in which one of the main results is that HPT holds for every class of finite structures of bounded treewidth; another result is that HPT holds for the class of all planar graphs.

At the Tarski Centenary Conference held in Warsaw in 2001, Johann A. Makowsky presented a survey of applications of another kind of preservation result that goes under the heading of the Feferman-Vaught Theorem 31. What Vaught and I had shown in our joint 1959 paper [16] was that for a great variety of sum and product operations $\mathrm{O}$ on structures $M_{i}(i \in I)$, the first-order properties of

$$
M=O\left\langle M_{i} \mid i \in I\right\rangle
$$

are determined by the first order properties of each $M_{i}$ together with the monadic secondorder properties of a structure on the index set $I$. It follows that elementary equivalence between structures is preserved under such operations $O$. In later work, Läuchli, Gurevich and Shelah extended our reduction-to-factors theorem to monadic second-order properties. In his paper, Makowsky gives a unified presentation of this work with emphasis on its algorithmic applications, in particular to splitting theorems for graph polynomials. I'll have to leave it at that, since it would take too much time to try to go further into that here.

The final thing I want to tell something about is the connection of Tarski's ideas and work with database theory. Here it is not a matter of direct influence but rather of the pervasiveness of his approach to things, since the development of database theory apparently proceeded quite independently. Jan Van den Bussche has written an excellent survey [46] of the connections, which I urge you to read; here are a few of the high points. Codd [8] introduced a relational algebra for expressing a class of generic (i.e., isomorphism invariant) queries on databases; he also proved that the queries expressible in his relational algebra are exactly those that are domain independent and definable in FO logic. For those who know Tarski's work on relation algebra, cylindric algebras and algebraic logic more generally, the immediate question to raise is the nature of the connection. ${ }^{15}$ (You can find a quick introduction to Tarski's work in this respect in Interlude VI of our biography [14. ${ }^{16}$ ) I view Tarski's work on algebraic logic as part of his general effort to reformulate logic in mathematical as opposed to metamathematical terms, in the hopes of thus making logic of greater mathematical interest. Tarski had done much work in the 1930s on Boolean algebras,

\footnotetext{
${ }^{14}$ According to Rossman, the implication was known to hold prior to his discovery.

${ }^{15}$ According to Van den Bussche (personal communication) the first people from the database community to recognize the connection between Codd's relational algebra and Tarski's cylindric algebras were Witold Lipski and his student Tomasz Imielinski, in a talk given at the very first edition of PODS (the ACM Symposium on Principles of Database Systems), held in Los Angeles, March 29-31, 1982. Their work was later published in Imielinski and Lipski [27.

${ }^{16}$ Some other applications to computer science-not discussed here-of Tarski's work on relation algebra are indicated on p. 339 and its footnote 4 of that interlude.
} 
of which algebras of sets and algebras of propositions (up to equivalence) are specific cases. Stone's representation theorem for Boolean algebras showed that every abstract BA is isomorphic to a concrete one in the sense of fields of sets, and in that sense the equational axioms of BA are complete. With Tarski's 1941 paper "On the calculus of relations" 38 he single-handedly revived and advanced the $19^{\text {th }}$ century work on binary relations by Peirce and Schröder, and introduced an elegant finite equational axiom system for relation algebra, from which all known special cases of valid relational identities could be deduced. However, it was shown by Roger Lyndon in 1950 that there are non-representable relation algebras, so Tarski's axioms are not complete; later, Donald Monk proved (in 1964) that there is no finite axiomatization of the valid equations in the language of these algebras. This is related to the fact that what can be expressed in relation algebra is exactly what can be expressed about binary predicates in 3 -variable FOL. ${ }^{17}$ See 30] for more on the history of relation algebras.

Given the weakness of relation algebra, in the early 1950s Tarski introduced the idea of cylindric algebras (CAs) of dimension $k$ for any $k \geq 2$, finite or infinite. (NB: Cylindric Algebras have nothing to do with Cylindrical Algebraic Decomposition.) In addition to the Boolean operations, these algebras use operations $C_{n}$ of cylindrification for each $n<k$ and diagonal constants $d_{n, m}$ for each $n, m<k$. The concrete interpretations are given by fields of subsets of a $k$-ary space $U^{k}$, with the $C_{n}$ interpreted as cylindrification along the $n$th axis, and the $d_{n, m}$ as the set of $k$-tuples in $U$ for which the $n$th and $m$ th terms are equal. Thus $k$ dimensional CAs abstract $k$-variable FOL with identity. The theory of CAs was extensively developed by students and colleagues of Tarski and the results are exposited in the volumes by Henkin, Monk and Tarski 24, 25]. It turns out that there are non-representable CAs for every $k \geq 2$, finite or infinite, but Henkin and Tarski showed that "locally finite" CAs are representable for every infinite $k$. A CA is called locally finite if for each element $a$ of the algebra, $C_{n}(a)=a$ for all but a finite number of $n<k$. The local-finiteness condition corresponds to each formula in FOL having at most a finite number of free variables, and the representation theorem for infinite dimensional locally finite CAs corresponds to the completeness theorem for FOL with identity.

As Van den Bussche points out in [46, the language of $\omega$-CAs provides an alternative to Codd's relational query language, and that of $k$-CAs for $k$ finite is an alternative to queries definable in FOL with at most $k$ distinct variables. But how does Codd's language match up with that of Relation Algebras (RAs)? In 43 it is shown that adjunction of a suitable "pairing axiom" to RA makes it as strong as FOL. It turns out that the corresponding idea has been developed in the case of database theory by Gyssens, Saxton and Van Gucht [22] using "tagging" operations, giving a form $\mathrm{RA}^{=}$that simulates Codd's relational algebra. ${ }^{18}$

So, does that justify John Etchemendy's statement that the shiny Oracle towers on Hwy 101 wouldn't be there without Tarski's recursive definition of satisfaction and truth? It would be more accurate to say that the Oracle towers wouldn't be there without the theoretical development of database theory, and that wouldn't be there without rethinking the model theory of first-order logic in relation-algebraic and/or cylindric-algebraic terms, and that wouldn't be there without Tarski's promotion of both model theory and algebraic logic. Does Larry Ellison know who Tarski is or anything about his work? At the time of my lecture, I wondered whether Ellison even knew who Codd was or the whole

\footnotetext{
${ }^{17} \mathrm{Cf}$. the papers [18, 19] dealing with expressibility/inexpressibility in Tarski's algebraic framework.

${ }^{18}$ Van den Bussche's article concludes with a survey of some interesting connections to constraint databases and geometric databases.
} 
theoretical development of database theory, without which the Oracle towers would indeed not be there. I learned subsequently from Jan Van den Bussche that not only did Ellison know about Codd's work but he marks the reading of Codd's seminal paper as the starting point leading to the Oracle Corporation; cf. his biography given by the "Academy of Achievement" at http://www.achievement.org/autodoc/page/ellobio-1/. Actually, Codd himself didn't refer to Tarski in his fundamental papers on database theory. But other workers in the subject, such as Imielinski and Lipski, and later, Kanellakis, did; they were well aware of the connection and brought explicit attention to it (cf., e.g. 28, p. 1085). In whatever way the claim is formulated, $I$ think it is fair to say that Tarski's ideas and the approaches he promoted are so pervasive that even if his influence in this and the various other areas of computer science about which I spoke was not direct it was there at the base, and-to mix a metaphor-it was there in the air, and so the nature and importance of his influence eminently deserves to be recognized.

\section{REFERENCES}

[1] M. Ajtai and Y. Gurevich. Datalog vs. first-order logic. J. Comput. System Sci., 49:562-588, 1994.

[2] A. Atserias, A. Dawar, and Ph. Kolaitis. On preservation under homomorphisms and unions of conjunctive queries. In 23rd ACM SIGMOD-SIGACT-SIGART Symposium on Principles of Database Systems (PODS), pages 319-329. ACM, 2004.

[3] Jon Barwise and Solomon Feferman, editors. Model-theoretic Logics. Springer-Verlag, 1985.

[4] M. Ben-Or, D. Kozen, and J. H. Reif. The complexity of elementary algebra and geometry. J. Comput. Syst. Sci., 32(2):251-264, 1986.

[5] Tevfik Bultan, Richard Gerber, and William Pugh. Symbolic model checking of infinite state systems using Presburger arithmetic. In CAV '97: Proceedings of the 9th International Conference on Computer Aided Verification, pages 400-411, London, UK, 1997. Springer-Verlag.

[6] John F. Canny. Some algebraic and geometric computations in PSPACE. In ACM Symposium on Theory of Computing, pages 460-467. ACM, 1988.

[7] B. F. Caviness and J. R. Johnson, editors. Quantifier Elimination and Cylindrical Algebraic Decomposition. Springer Series in Symbolic Computation. Springer-Verlag, 1998.

[8] E. F. Codd. A relational model of data for large shared data banks. Comm. ACM, 13(6):377-387, 1970.

[9] George E. Collins. Quantifier elimination for real closed fields by cylindrical algebraic decompositionpreliminary report. SIGSAM Bulletin, 8(3):80-90, August 1974.

[10] George E. Collins. Quantifier elimination for real closed fields by cylindrical algebraic decomposition. In Proceedings Second GI Conference on Automata Theory and Formal Languages, pages 134-183, Berlin, 1975. Lecture Notes in Computer Science 33, Springer-Verlag. Reprinted in [7], pp. 85-121.

[11] George E. Collins and Hoon Hong. Partial cylindrical algebraic decomposition for quantifier elimination. Journal of Symbolic Computation, 12(3):299-328, 1991. Reprinted in 7], pp. 174-200.

[12] M. Davis. The prehistory and early history of automated deduction. In J. Siekmann and G. Wrightson, editors, Automation of Reasoning 1: Classical Papers on Computational Logic 1957-1966, pages 1-28. Springer, Berlin, Heidelberg, 1983.

[13] Heinz-Dieter Ebbinghaus and Jörg Flum. Finite Model Theory. Springer-Verlag, Berlin, Heidelberg, New York, second edition, 1991.

[14] Anita Burdman Feferman and Solomon Feferman. Alfred Tarski: Life and Logic. Cambridge University Press, New York, NY, USA, 2004.

[15] Solomon Feferman. Tarski's conceptual analysis of semantical notions. In A. Benmakhlouf, editor, Sémantique et épistémologie, pages 79-108. Editions Le Fennec, Casablanca [distrib. J. Vrin, Paris], 2004.

[16] Solomon Feferman and Robert L. Vaught. The first order properties of products of algebraic systems. Fundamenta Mathematicae, 47:57-103, 1959.

[17] Michael J. Fischer and Michael O. Rabin. Super-exponential complexity of Presburger arithmetic. In R. Karp, editor, Complexity of Computation, volume 7 of SIAM-AMS Proceedings, pages 27-42, Providence, R.I., 1974. American Mathematical Society. Reprinted in [7], pp. 122-135. 
[18] Andrea Formisano, E. G.Omodeo, and A. Policriti. Three-variable statements of set-pairing. Theoretical Computer Science, 322(1):147-173, 2004.

[19] Andrea Formisano, E. G.Omodeo, and A. Policriti. The axiom of elementary sets on the edge of Peircean expressibility. Journal of Symbolic Logic, 70:953-968, 2005.

[20] Laurent Fribourg and Hans Olsén. Proving safety properties of infinite state systems by compilation into Presburger arithmetic. In Antoni W. Mazurkiewicz and Józef Winkowski, editors, CONCUR, volume 1243 of Lecture Notes in Computer Science, pages 213-227. Springer, 1997.

[21] G. Gierz, K. H. Hoffman, K. Keimel, J. D. Lawson, M. Mislove, and Dana S. Scott. Continuous Lattices and Domains. Encyclopedia of Mathematics and its Applications, 93. Cambridge University Press, Cambridge, England, 2003.

[22] M. Gyssens, L. V. Saxton, and D. Van Gucht. Tagging as an alternative to object creation. In J. C. Freytag, D. Maier, and G. Vossen, editors, Query Processing for Advanced Database Systems, pages 201-242. Kaufmann, San Mateo, 1994.

[23] Paul Halmos. I Want to be a Mathematician: an Automathography. Springer-Verlag, Berlin, Heidelberg, New York, 1985.

[24] Leon Henkin, Donald Monk, and Alfred Tarski. Cylindric Algebras, volume 1. North-Holland, Amsterdam, 1971.

[25] Leon Henkin, Donald Monk, and Alfred Tarski. Cylindric Algebras, volume 2. North-Holland, Amsterdam, 1971.

[26] Wilfrid Hodges. Truth in a structure. Proceedings of the Aristotelian Society, 86:131-151, 1985/86.

[27] Tomasz Imielinski and Witold Lipski, Jr. The relational model of data and cylindric algebras. Journal of Computer and System Sciences, 28(1):80-102, 1984.

[28] Paris C. Kanellakis. Elements of relational database theory. In Jan van Leeuwen, editor, Handbook of Theoretical Computer Science, Volume B: Formal Models and Sematics (B), pages 1073-1156. MIT Press, 1990.

[29] J. L. Lassez, V. L. Nguyen, and E. A. Sonnenberg. Fixed point theorems and semantics: a folk tale. Information Processing Letters, 14:112-116, 1982.

[30] R. D. Maddux. The origin of relation algebras in the development and axiomatization of the calculus of relations. Studia Logica, 50(3/4):421-455, 1991.

[31] Johann A. Makowksy. Algorithmic uses of the Feferman-Vaught theorem. Annals of Pure and Applied Logic, 126:159-213, 2004.

[32] Derek C. Oppen. A $2^{2^{2^{p n}}}$ upper bound on the complexity of Presburger arithmetic. Journal of Computer and System Sciences, 16:323-332, 1978.

[33] Mojzesz Presburger. über die Vollständigkeit eines gewissen Systems der Arithmetik ganzer Zahlen, in welchem die Addition als einzige Operation hervortritt. Comptes Rendus du I congrs des Mathmaticiens des Pays Slaves, pages 92-101, 1929.

[34] Eric Rosen. Some aspects of model theory and finite structures. Bulletin of Symbolic Logic, 8:380-403, 2002.

[35] Benjamin Rossman. Existential positive types and preservation under homomorphisisms. In Prakash Panangaden, editor, Proceedings of the Twentieth Annual IEEE Symp. on Logic in Computer Science, LICS 2005, pages 467-476. IEEE Computer Society Press, June 2005.

[36] Dana S. Scott. Data types as lattices. SIAM Journal on Computing, 5:522-587, 1976.

[37] N. Shankar. Little engines of proof. In L.-H. Eriksson and P. Lindsay, editors, FME 2002: Formal Methods - Getting IT Right, Copenhagen, pages 1-20. Springer-Verlag, 2002.

[38] Alfred Tarski. On the calculus of relations. Journal of Symbolic Logic, 6:73-89, 1941.

[39] Alfred Tarski. A Decision Method for Elementary Algebra and Geometry. University of California Press, second edition, 1951. Reprinted in 7], pp. 24-84.

[40] Alfred Tarski. A lattice-theoretical fixpoint theorem and its applications. Pacific Journal of Mathematics, 5:285-309, 1955.

[41] Alfred Tarski. The concept of truth in formalized languages. In John Corcoran, editor, Logic, Semantics, Metamathematics. Papers from 1923 to 1938, pages 182-278. Oxford University Press, second edition, 1983. Original date, 1935.

[42] Alfred Tarski. On definable sets of real numbers. In John Corcoran, editor, Logic, Semantics, Metamathematics. Papers from 1923 to 1938, pages 110-142. Oxford University Press, second edition, 1983. Original date, 1931. 
[43] Alfred Tarski and Steven Givant. A Formalization of Set Theory without Variables. Number 41 in Colloquium Publications. American Math. Society, Providence, 1987.

[44] Alfred Tarski and Steven Givant. Tarski's system of geometry. Bulletin of Symbolic Logic, 5:175-214, 1999.

[45] Alfred Tarski and Robert L. Vaught. Arithmetical extensions of relational systems. Compositio Mathematica, 13:81-102, 1957.

[46] Jan Van den Bussche. Applications of Alfred Tarski's ideas in database theory. Lecture Notes in Computer Science, 2142:20-37, 2001.

This work is licensed under the Creative Commons Attribution-NoDerivs License. To view a copy of this license, visit http://creativecommons.org/licenses/by-nd/2.0/ or send a letter to Creative Commons, 559 Nathan Abbott Way, Stanford, California 94305, USA. 